\section{P63 ESTABLISHING THE COST OF HOSPITALISED COMMUNITY ACQUIRED PNEUMONIA (CAP): A HOSPITAL EPISODE STATISTICS (HES) ANALYSIS}

${ }^{1} \mathrm{D}$ Jones, ${ }^{1} \mathrm{~J}$ Campling, ${ }^{1} \mathrm{G}$ Ellsbury, ${ }^{1} \mathrm{C}$ Czudek, ${ }^{1} \mathrm{H}$ Madhava, ${ }^{2} \mathrm{M}$ Slack. ${ }^{1}$ Pfizer Ltd, Tadworth, UKi ${ }^{2}$ Griffith University, Southport QLD 4215, Australia

\subsection{6/thoraxjnl-2017-210983.205}

Introduction There are two types of pneumococcal vaccine available for adults: polysaccharide vaccine (PPV23) and a conjugate vaccine (PCV13). PCV13 vaccination is efficacious in adults aged 65 and over at preventing both invasive pneumococcal disease (IPD) and pneumonia caused by the serotypes in the vaccine ${ }^{1}$ while the evidence is inconsistent for PPV23.,3 The Joint Committee on Vaccination and Immunisation (JCVI) concluded that vaccination of $\geq 65$ years with PCV13 was not cost-effective, and recommended against a national immunisation programme. As part of this analysis, a pneumonia admission (ICD-10 code J18) was costed at $£ 715^{4}$

Aim To obtain an alternative estimate of the cost of a hospitalised CAP both during the acute admission and following discharge.

Materials and Methods All patients aged $\geq 65$ years with ICD10 J18 registered in HES between April 1 st 2014 and March 31 st 2015 were identified and their hospital-based activity tracked for 12 months. All in-patient, out-patient, and $\mathrm{A}$ and $\mathrm{E}$ attendances for these patients were extracted and the overall volumes and costs of these activities assessed over various timeframes. Costs were derived from the tariff via the Healthcare Resource Group codes.

Results The average cost of the initial in-patient (aged $\geq 65$ years) admission for pneumonia (J18) was estimated at $£ 3256$. Over the 1-90 day period following the initial admission $69 \%$ of patients registered some additional utilisation of health care at an average cost of $£ 2090$.

Conclusion It is important that any cost effectiveness assessment accurately captures the costs averted by the intervention. Our analysis suggests that the cost of a pneumonia admission (J18) is 4-fold higher than that utilised in the 2016 analysis. In addition, significant additional costs may result from exacerbation of any underlying co-morbidities, thereby increasing the cost associated with a CAP infection. Even if only some of these additional costs were due to the original CAP infection, the value used in the original analysis significantly underestimated the cost of CAP.

Please refer to page A258 for declarations of interest in relation to abstract P63.

\section{REFERENCES}

1. Bonten MJM, et al. N Engl I Med 2015;372:1114-25.

2. Moberley S, et al. Cochrane Database of Systematic Reviews 2013.

3. Tin Tin Htar M, et al. PLOS ONE 2017;12(5):e0177985.

4. Van Hoek AJ, et al. PLOS ONE 2016;11(2):e0149540.

\section{P64 \\ PREDICTING THE IMPACT OF TOBACCO PRICE INCREASE POLICIES ON COPD BURDEN IN ITALY}

${ }^{1} \mathrm{~V}$ Jani, ${ }^{1} \mathrm{~L}$ Potts, ${ }^{2} \mathrm{G}$ Pesce, ${ }^{2} \mathrm{~A}$ Marcon, ${ }^{2} \mathrm{~S}$ Accordini, ${ }^{1} \mathrm{D}$ Jarvis, ${ }^{1} \mathrm{C}$ Minelli. ${ }^{1}$ Imperial College London, London, UK; ${ }^{2}$ University of Verona, Verona, Italy

\subsection{6/thoraxjnl-2017-210983.206}

Introduction and Objective Smoking has a large health and economic burden on populations, much of which is through increased risk of COPD, a major cause of disease, disability and death. COPD is set to rise with population ageing, and smoking initiation rates are increasing in young adolescents across Europe. We used Health Impact Assessment (HIA) analysis to predict the potential impact of tobacco price increase policies, to which youngsters are particularly sensitive, on future COPD burden in Italy, a country with low tobacco price relative to the rest of Europe.

Methods As part of the Ageing Lungs in European Cohorts (ALEC) project (www.alecstudy.org), we used DYNAMO-HIA for a Markov-based modelling approach to HIA analysis. Demographic and smoking data from the Italian population were used, together with data on the effects of smoking and health burden of COPD. A 'maximum' and a 'realistic' scenario were simulated to reflect different price increase policies: a $138 \%$ increase to match UK price (highest in Europe), and a $50 \%$ increase. Using published figures for price elasticities, we simulated changes to smoking behaviours over a 40 year period, evaluated their effects on COPD burden, and compared the two scenarios to a 'business as usual' scenario.

Results The projected population pyramid confirmed Italy as an ageing population with increasing COPD burden. Over the 40 year period, the maximum scenario showed reduction in smoking prevalence mainly through an increase in never smokers. Compared with 'business as usual', this translated in a substantial decrease in COPD incidence and prevalence, with consequent reduction in mortality and increase in average life expectancy. The realistic scenario showed effects of smaller magnitude in the same direction.

Conclusions Tobacco price increase policies would be effective in reducing future COPD burden in Italy. To provide a wider European perspective, we are now extending this work to countries with different smoking behaviours and tobacco prices.

\section{P65 QUALITY OF INPATIENT CARE FOR COPD EXACERBATIONS AND IT'S IMPACT ON CLINICAL OUTCOMES}

B Cushen, A Alsaid, E Cleere, P MacHale, L Tompkins, I Sulaiman, G Greene, E MacHale, RW Costello. Royal College of Surgeons, Dublin, Ireland

\subsection{6/thoraxjnl-2017-210983.207}

High rates of rehospitalisation in the 90 days following COPD exacerbation are a concern internationally due to their unpredictable nature, the impact on patient's health and the pressures they pose on healthcare systems. Strategies to reduce rehospitalisation have looked to improve inpatient management at the time of the index admission. We assessed the rate of adherence to international acute COPD management guidelines and examined which components of these guidelines have the greatest impact on clinical outcomes. Data from 208 patients hospitalised with an acute exacerbation of COPD was retrospectively collected from the medical chart. Adherence to five key components of COPD management was assessed. These included 1) Arterial blood gas measurement, 2) Administration of Controlled Oxygen therapy, 3) Regular short-acting bronchodilator therapy, 4) Prescription of systemic steroids (oral if suitable) and 5) Prescription of appropriate antibiotics, where applicable. Hospital length of stay(LOS) and readmissions up to 90 days following discharge were recorded. The mean age was 71 years and the majority were female. The mean $\mathrm{FEV}_{1}$ was $48 \%$ predicted and the median DECAF score was 1 (2) suggesting a low risk exacerbation. Almost 50\% had a co-existent consolidation on chest radiograph. The median 